Forschungszentrum Karlsruhe

Technik und Umwelt

Wissenschaftliche Berichte

FZKA 6071

\title{
A Compact Detector for the Measurement of the Cosmic Ray Muon Charge Ratio
}

B. Vulpescu, J. Wentz, I. M. Brâncuş, H. Rebel, A. F. Badea, H. Bozdog, M. Duma, A. Haungs, H.-J. Mathes, M. Petcu, M. Roth Institut für Kernpyhsik 



\section{ForschungSzentRUM KaRlSRUhe \\ Technik und Umwelt \\ Wissenschaftliche Berichte \\ FZKA 6071}

\section{A compact detector for the measurement of the cosmic ray muon charge ratio}

B. Vulpescu ${ }^{1,2}$, J. Wentz, I.M. Brâncuşs ${ }^{1}$, H. Rebel, A.F. Badea ${ }^{1}$, H. Bozdog ${ }^{1}$, M. Duma ${ }^{1}$, A. Haungs, H.-J. Mathes, M. Petcu ${ }^{1}$ and M. Roth

\section{Institut für Kernphysik}

1 NIPNE, P.O.B. MG-6, RO-7690 Bucharest, Romania

2 Present address: Forschungszentrum Karlsruhe, Institut für Kernphysik, Postfach 3640, D - 76021 Karlsruhe, Germany

Forschungszentrum Karlsruhe GmbH, Karlsruhe 1998 
Als Manuskript gedruckt

Für diesen Bericht behalten wir uns alle Rechte vor

Forschungszentrum Karlsruhe GmbH

Postfach 3640, 76021 Karlsruhe

Mitglied der Hermann von Helmholtz-Gemeinschaft

Deutscher Forschungszentren (HGF)

ISSN 0947-8620 


\section{Abstract}

A compact sampling calorimeter is developed and used to measure the ratio between positive and negative muons in the cosmic ray flux at momenta below $1 \mathrm{GeV} / \mathrm{c}$. The delayed coincidence method is used, based on the reduced mean life time of negative muons due to nuclear capture in matter. It is shown that aluminum is a good choice as capture medium for negative muons and suitable for a simple detector set-up. The muon charge ratio is found to be $1.30 \pm 0.05$ for a mean momentum of $0.86 \mathrm{GeV} / \mathrm{c}$ and over a zenith angle acceptance with $\Theta_{\text {mean }}=26^{\circ}$.

\section{Zusammenfassung}

\section{Ein kompakter Detektor zur Messung des Ladungsverhältnisses von Myonen aus der Höhenstrahlung}

Zur Messung des Ladungsverhältnisses von positiven zu negativen Myonen aus der Höhenstrahlung für Impulse kleiner $1 \mathrm{GeV} / \mathrm{c}$ wird ein kompaktes Sampling - Kalorimeter entwickelt und aufgebaut. Basierend auf der Änderung der mittleren Lebenszeit von negativen Myonen in Materie durch nuklearen Einfang wird eine verbesserte "delayed coincidence" - Methode benutzt. Es wird gezeigt, daß Aluminium als Einfangmedium für negative Myonen geignet ist um eine kompakte Auslegung des Detektors zu ermöglichen. Das Ladungsverhältnis der Myonen bei einem mittleren Impuls von $0.86 \mathrm{GeV}$ und einem mittleren Zenitwinkel von $26^{\circ}$ wird zu $1.30 \pm 0.05$ bestimmt. 


\section{Contents}

1 Introduction 1

2 The method $\quad 3$

3 The apparatus $\quad 4$

3.1 Electronic layout and data acquisition . . . . . . . . . . . . 6

3.2 Detector efficiency calculations . . . . . . . . . . . . . . 8

3.2.1 Energy calibration of the active layers . . . . . . . . . . . 9

3.2.2 Energy deposit thresholds for the MTDC events . . . . . . . . . . . 10

4 Discussion and results $\quad 11$

5 Conclusions $\quad 14$

$\begin{array}{llr}6 & \text { Appendix } & 18\end{array}$ 


\section{Introduction}

The ratio of positive to negative cosmic ray muons is of great interest for different aspects of elementary particle- and astrophysics. At high energy, the muon charge ratio reflects specific features of hadronic interaction $[1,2,3]$. Models of cosmic ray interaction in the atmosphere are used to interpret data from extensive air shower experiments, aiming at information about the energy spectrum and chemical composition of the primary flux. As indicated by high-energy experiments, the observed positive charge excess increases slightly with the muon energy and can be correlated with enhanced strangeness production, favoring $K^{+}$and $K^{0}$ as compared to $K^{-}$. The low energy muon flux is the bulk source of $\nu_{e}, \bar{\nu}_{e}, \nu_{\mu}$ and $\bar{\nu}_{\mu}$, the ratios $\nu_{e} / \bar{\nu}_{e}$ and $\nu_{\mu} / \bar{\nu}_{\mu}$ being directly related to the muon charge ratio. Correct interpretation of the experiments investigating the ratio $\nu_{e}+\bar{\nu}_{e}$ to $\nu_{\mu}+\bar{\nu}_{\mu}$ needs precise knowledge of the contribution of each neutrino flavor, since the detection efficiency is rather different for neutrinos and antineutrinos $[4,5,6]$.

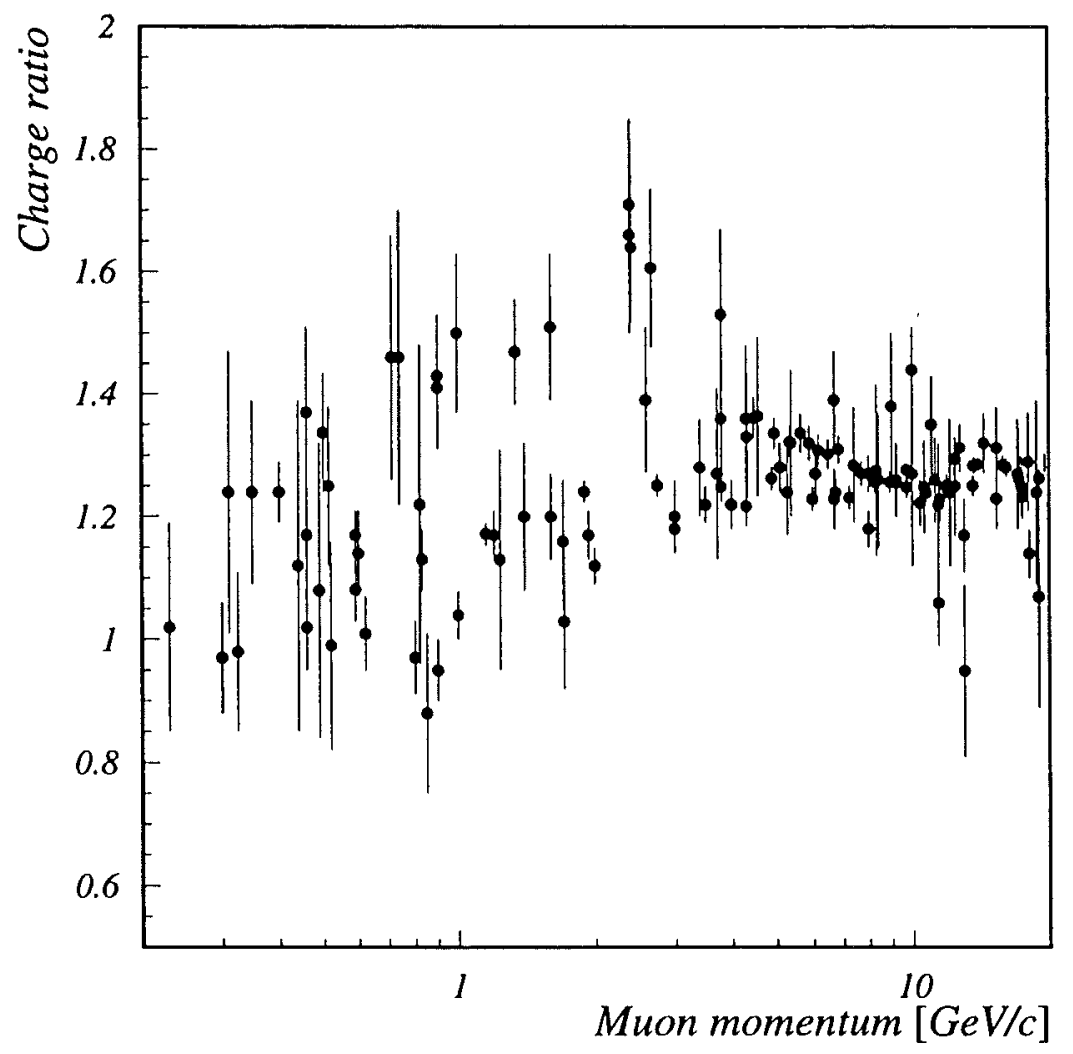

Figure 1: Compilation of results on the charge ratio of vertical muons in the momentum range up to $20 \mathrm{GeV} / \mathrm{c}$. Experimental data - see Appendix.

Several experimental techniques have been applied to measure the muon charge ratio. Fig. 1 compiles results up to $20 \mathrm{GeV} / \mathrm{c}$ muon momentum, but there are also measurements in a higher momentum range up to $10^{5} \mathrm{GeV} / \mathrm{c}$. While around $10 \mathrm{GeV} / \mathrm{c}$ the data 
appear in fair agreement, the picture is not clear around $1 \mathrm{GeV} / \mathrm{c}$. In most experiments the separation of positive and negative particles was performed by means of a magnetic field, historically first by solid iron magnetic lenses, or by combining magnets with a cloud chamber [7]. Later on magnetic spectrometer set-ups became the most common instruments to measure charged particle spectra. Different types of detectors were used for refined methods for particle identification: drift-tube tracking hodoscopes, time-of-flight systems, transition-radiation detectors, electromagnetic calorimeters, Cherenkov threshold detectors etc. In the output signals of such detectors, cuts are made to separate muons from other cosmic ray particles, especially from low-energy electrons. Compact set-ups have been used in balloon-borne experiments to extend the measurements to different altitudes in the atmosphere (MASS2 [8], IMAX [9] or HEAT [10]). The scattered results in the range below a few GeV/c in Fig. 1 indicate experimental uncertainties. Spectrometers are difficult to use for very low momenta, due to the large percentage of electrons present in the secondary cosmic rays and different acceptances for positive and negative particles.

A different experimental approach is the method of delayed coincidences $[11,12]$ in which the muons are identified via their decay by recording a delayed coincidence between the incoming muon, stopped in some absorber, and the electron emerging from the decay. The separation between positive and negative muons is done by using two different absorbers, one with low atomic number (graphite or sulphur) and one with high atomic number (lead). In the light absorber negative muons are considered to decay with the same mean life time as the positive muons, while in the heavy one they are captured in muonic atom orbits and disappear by a nuclear capture. The atomic and nuclear capture processes of negative muons have been theoretically studied in detail [13] and the capture rates have been measured for almost all elements and for some of their isotopes [14]. Correct capture rates and mean life times have been considered in two experiments using a single absorber of large volume: KAMIOKANDE [15] and KARMEN [16, 17]. These devices are neutrino detectors with facilities for vertex reconstruction and time measurements. Measurements with KAMIOKANDE, a $2140 \mathrm{t}$ pure water Cherenkov detector placed $1000 \mathrm{~m}$ underground, reported a muon charge ratio of $1.37 \pm 0.06$ at $1.2 \mathrm{TeV} / \mathrm{c}$. The KARMEN detector is a $56 \mathrm{~m}^{3}$ liquid scintillator and measured $1.28 \pm 0.02$ at $5.1 \mathrm{GeV} / \mathrm{c}$ [17]. In these experiments the difference between positive and negative muons is determined by a precise analysis of the decay time curves of negative muons due to the capture in oxygen and carbon nuclei, respectively. The mean life time of negative muons in various materials is given in Tab. 1. It can be noticed that the difference to the mean life time of the free decay of positive muons is still small for carbon or oxygen, thus one needs high statistical accuracy for a good separation.

In the present experiment aluminum is used as absorber to separate positive from negative muons. The mean life time of negative muons is less than half of the mean life time for positive muons, but still measurable with simple electronics and at a reasonable decay rate (see Tab. 1). The aluminum absorber is arranged as several passive layers, 
alternating with active detector modules. This configuration improves the collection rate against re-absorption of decay electrons coming from muons stopped in aluminum.

Table 1: Total capture data for various elements [14]

\begin{tabular}{lcc}
\hline & Mean life time (ns) & Total capture probability (\%) \\
\hline Vacuum & $2197.03 \pm 0.04$ & 0.00 \\
Carbon & $2026.3 \pm 1.5$ & 7.85 \\
Oxygen & $1795.4 \pm 2$. & 18.43 \\
Sodium & $1204.0 \pm 2$. & 45.39 \\
Magnesium & $1067.2 \pm 2$. & 51.66 \\
Aluminum & $864.0 \pm 1$. & 60.95 \\
Silicon & $756.0 \pm 1$. & 65.86 \\
Phosphor & $611.2 \pm 1$. & 72.43 \\
Sulphur & $554.7 \pm 1$. & 75.00 \\
Lead & $75.4 \pm 1$. & 97.25 \\
\hline
\end{tabular}

\section{The method}

The main idea of the delayed coincidence method exploits the different behavior of negative and positive muons after they are brought to rest in matter. While the positive muon has only the possibility to decay, the negative muon is captured by the host atom forming a muonic atom. Within a short time $\left(10^{-13} \mathrm{~s}\right)$ the muon cascades to the lowest level $1 \mathrm{~s}$ and can undergo either decay or capture by the nucleus. The competition between the two processes reduces the mean life time of the decay and is characterized by

$$
\begin{aligned}
& \Lambda_{\text {total }}=\Lambda_{\text {capture }}+\Lambda_{\text {decay }} \\
& \Lambda_{\text {decay }}=Q \tau_{\mu^{+}}^{-1} \\
& \Lambda_{\text {total }}^{-1}=\tau_{\mu^{-}}<\tau_{\mu^{+}}=2.19703 \mu \mathrm{s} .
\end{aligned}
$$

The nuclear capture rate is determined by the overlap of the muonic orbit with the nucleus and it follows roughly a power law of the atomic number $\left(\Lambda_{\text {capture }} \sim Z^{4},[18]\right)$. The Huff factor $Q$ takes into account that the normal muon decay rate is slightly reduced due to the binding of the negative muon in the atomic orbit, shrinking the phase space for the decay. In compounds the atomic capture prefers the nucleus with the larger nuclear capture rate, i.e. larger atomic number. For instance, in a plastic scintillator base material (polyvinyl-toluol, $2-\mathrm{CH}_{3} \mathrm{C}_{6} \mathrm{H}_{4} \mathrm{CH}=\mathrm{CH}_{2}$ monomer) only the carbon capture is significant. 
The actual detector consists of three materials which contribute to the stopping of low energy muons: lead, aluminum and scintillator (carbon). Nuclear capture characteristics can be seen in Tab. 1. While the effective mean life time of the negative muon is too short and the decay probability too small in case of a lead absorber, carbon gives too little difference to the positive muon mean life time, but aluminum appears to be an optimum choice. Muonic atoms of aluminum have a quite different mean life time compared with the free decay of the positive muons combined with a reasonable decay probability. Aluminum is relatively cheap, available with a high degree of purity and easy to be shaped according to the experimental requirements. In the present experiment the decay time signals from the positive and negative muons stopped in the detector are collected in $\Delta t$ bins. The total muon decay curve is a superposition of four exponentials reflecting the decay of positive and negative muons stopped in different media. If $N_{+}$and $N_{-}$are the numbers of positive and negative muons which enter the detector, the decay curve is given by the sequence of the $\Delta N$ numbers of decay signals accumulated in time bins $\left(t_{i}, t_{i}+\Delta t\right)$, with the index $i$ running from $1\left(t_{1}=0\right)$ to the maximum allowed by the total observation time window

$$
\Delta N\left(t_{i}, t_{i}+\Delta t\right)=N_{+} c_{0} \delta_{0} \exp \left(-\frac{t_{i}}{\tau_{0}}\right)+N_{-}\left[\sum_{j=1}^{3} c_{j}^{*} \delta_{j} \exp \left(-\frac{t_{i}}{\tau_{j}}\right)\right] .
$$

There $\delta_{k}=\left[1-\exp \left(-\frac{\Delta t}{\tau_{k}}\right)\right], k=0,1,2,3$ are corrections due to integrated time signals over the bin width. Index 0 stands for positive muons, while $1,2,3$ correspond to the negative muons decaying in aluminum, lead and carbon, respectively. By a fit of eq. 4 to the experimental decay curve, $\mathrm{N}_{+}$and $\mathrm{N}_{-}$can be deduced. In order to reduce the number of free parameters, the detection probabilities for the decay electrons, $\mathrm{c}_{0}$ and $\mathrm{c}_{j}^{*}, j=1$, 2, 3, are determined by Monte-Carlo (MC) simulation with GEANT 3.21 [19]. Because this version of GEANT does not include nuclear capture, the detection probabilities for negative muons are split in

$$
c_{j}^{*}=c_{j} p_{\text {decay }}^{j} \quad j=1,2,3,
$$

where only $c_{j}$ have to be determined from the simulation and the decay probabilities $\mathrm{p}_{\text {decay }}^{j}$ are taken from [14]. Secondary particle production following the nuclear capture can be neglected because neutrons and gammas, evaporating from the highly excited nucleus, will hardly fake a decay electron.

\section{The apparatus}

The WILLI detector (Weakly Ionizing Lepton Lead Interaction) is installed at the National Institute of Nuclear Physics and Engineering - Horia Hulubei, Bucharest, $44^{\circ} 26^{\prime} \mathrm{N}$ latitude and $26^{\circ} 04^{\prime} \mathrm{E}$ longitude near sea level. It was originally designed for prototype studies of electromagnetic cascades induced by interaction of high energy cosmic ray 
muons in lead. The calorimeter was conceived like a sampling device, with 20 lead absorber plates of $1 \mathrm{~m}^{2}$ area and $1 \mathrm{~cm} \approx 2$ r.l. thickness alternating with plastic scintillators (see Fig. 2). The detector is placed in the basement of a building, under $\approx 60 \mathrm{~cm}$ of reinforced concrete. A $10 \mathrm{~cm}$ lead shield at the top of the instrument eliminates low energy electrons and gammas and sets the energy threshold of the detector. The $90 \times 90 \times 3 \mathrm{~cm}^{3}$ scintillator plates are housed in aluminum boxes with $1 \mathrm{~cm}$ thick bottom and lateral walls.

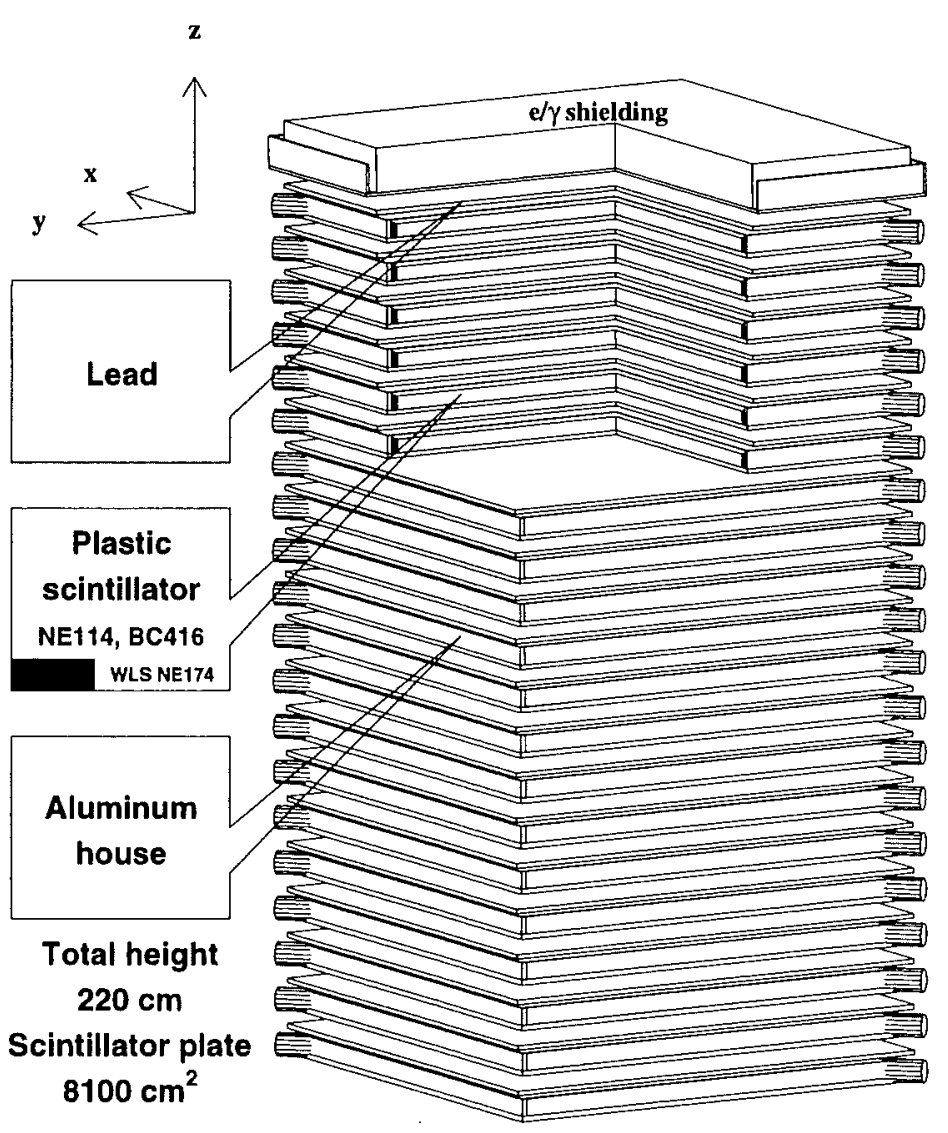

Figure 2: The WILLI detector.

Fig. 3 gives a horizontal cross section of a scintillator plate inside an aluminum box viewed from above. Because of the large scintillator area, a configuration with two photomultipliers (PM) placed in opposite corners is used to enhance light collecting. The light output is collected from the scintillator sides by wave length shifters (WLS), connected in pairs with plastic light guides placed in opposite corners. The scintillator, WLS and the light guides are wrapped in aluminum foil. All 20 modules form a stack of about $2 \mathrm{~m}$ height and the detector is placed in the basement of a building, under $\approx 60 \mathrm{~cm}$ of reinforced concrete, in a vertical position. 


\section{1, 2 - plastic light guides to photo-multipliers}

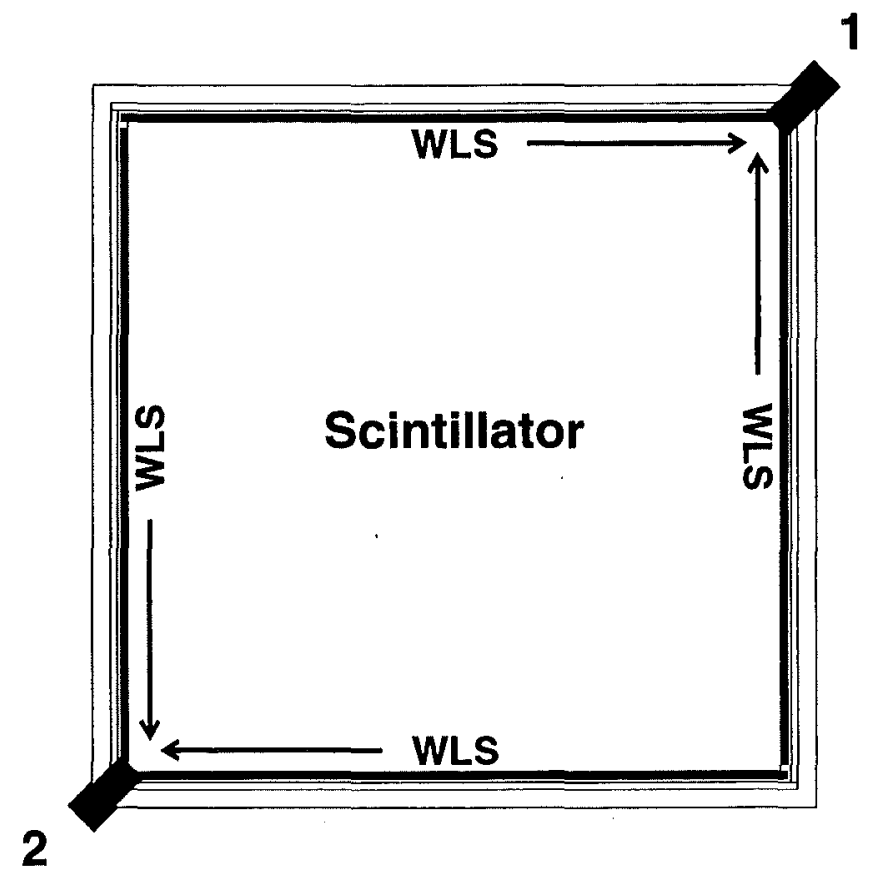

Figure 3: The active element.

Muons of low energy may stop in the detector and the eventual decay electrons are detected if they deposit energy over the threshold in one scintillator. A first trigger from the $1^{\text {st }}$ and the $4^{\text {th }}$ layer (counting from top) starts the time measurement, when a muon enters and the delayed signal from the decay electron is recorded in the 16 remaining layers.

\subsection{Electronic layout and data acquisition}

The data acquisition system is designed to give information about the energy deposit in all the 20 calorimeter layers and time information from the lowest 16 layers (see Fig. 4). Each PM supplies three analog signals: the first, A, is collected from the anode and is used for the energy measurement; a second signal, D, from the third dynode (counting from the anode side) is intended to enlarge, if necessary, the dynamic range towards high energy deposits in the scintillator; a third signal, $T$, from the first dynode is used for the time measurements. The maximum of the shaped anode pulse is detected in a "sample \& hold" module ( $\mathrm{S} \& \mathrm{H}$ ) and fed into a 12 bit ADC converter MPV906 (VME standard). For the main trigger, $\mathrm{T}_{\mathbf{1}}$, four channels are available in a coincidence scheme within $50 \mathrm{~ns}$. For recording the time history of one event a Multiple Time Digital Converter (MTDC) is used. It is interfaced with a VME bus by a WRAP-1/68K board using a TS68901 MultiFunction Peripheral chip (MFP901). The time signals are first compared with a reference 


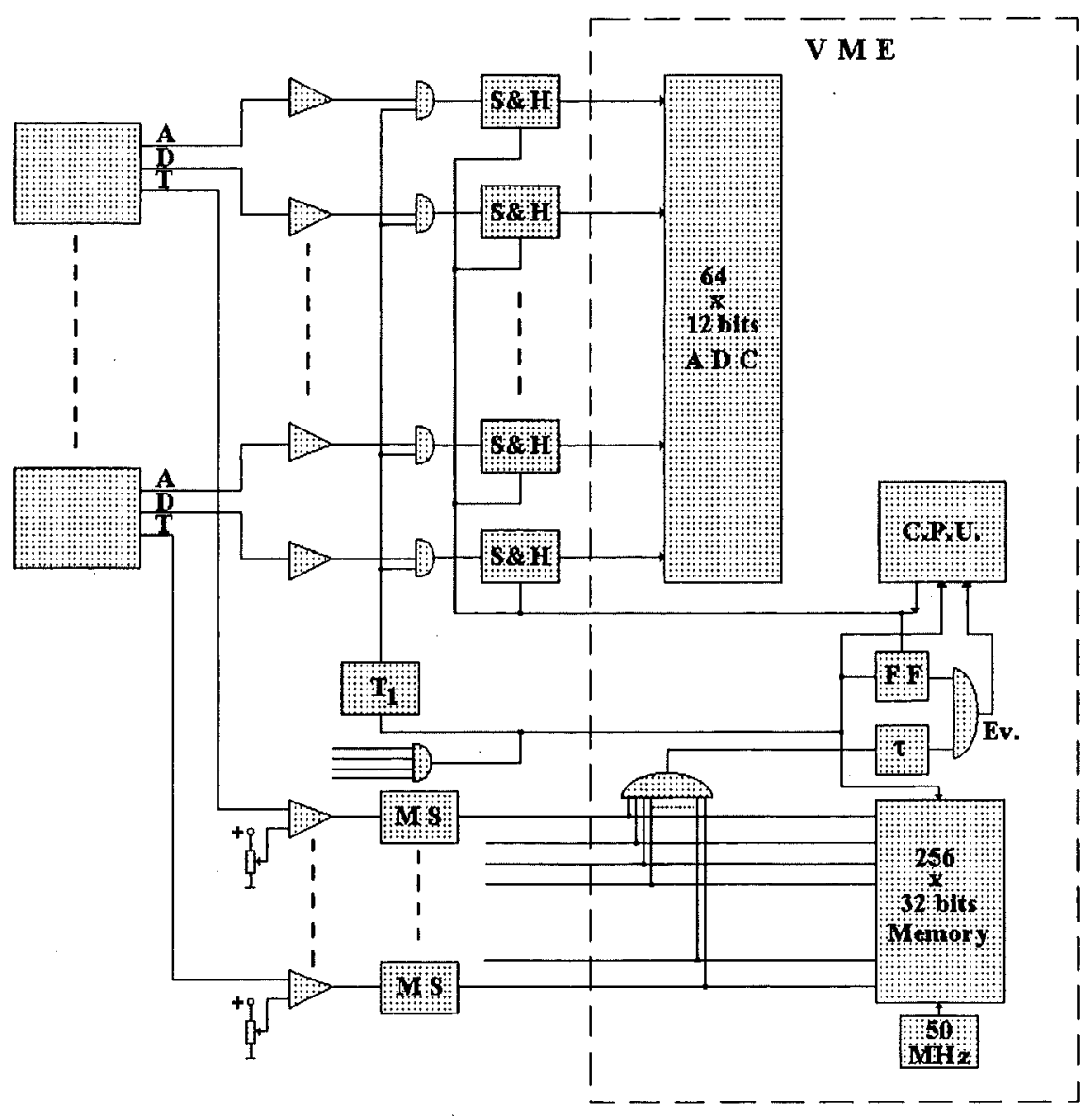

Figure 4: Electronic layout.

low voltage, which sets pulse amplitude thresholds on all 16 channels. The TTL signals are fed further to monostables MS which stretch the pulse length to $40 \mathrm{~ns}$.

All $16 \times 2$ time signals are stored in eight $256 \times 4$ bits RAM modules every 20 ns (with a $50 \mathrm{MHz}$ address counter). A second trigger is made by sampling the result after $80 \mathrm{~ns}(\tau)$ from the main trigger. An event data block will be written on the storage medium when both triggers are given. The expected background comes from uncorrelated particles, like a muon crossing the detector without stopping and another particle, either electron, gamma or another muon, entering the detector from the sides. Another source is the noise along the time electronic chains which can be partially reduced by adjusting the low voltage thresholds and asking for coincidence between both PM of each scintillator plate. The cuts made by the software are very restricting since the loss of some decay events is less important for the later analysis than the contamination with background events. Fig. 5 shows an individual event from the off-line computer event display, containing the ADC and MTDC information of the lowest 16 scintillator layers (32 PM). The continuous muon 
"track" is seen as zero time signals down to a "stopping layer" and the decay electron as a short delayed "track" in the vicinity of the "stopping layer" (in this case the electron is detected in the next active layer after 179 clock pulses, about $3.6 \mu$ s after the main trigger). Finally a histogram with 256 bins is built, which corresponds to the decay curve of muons stopped in the detector.

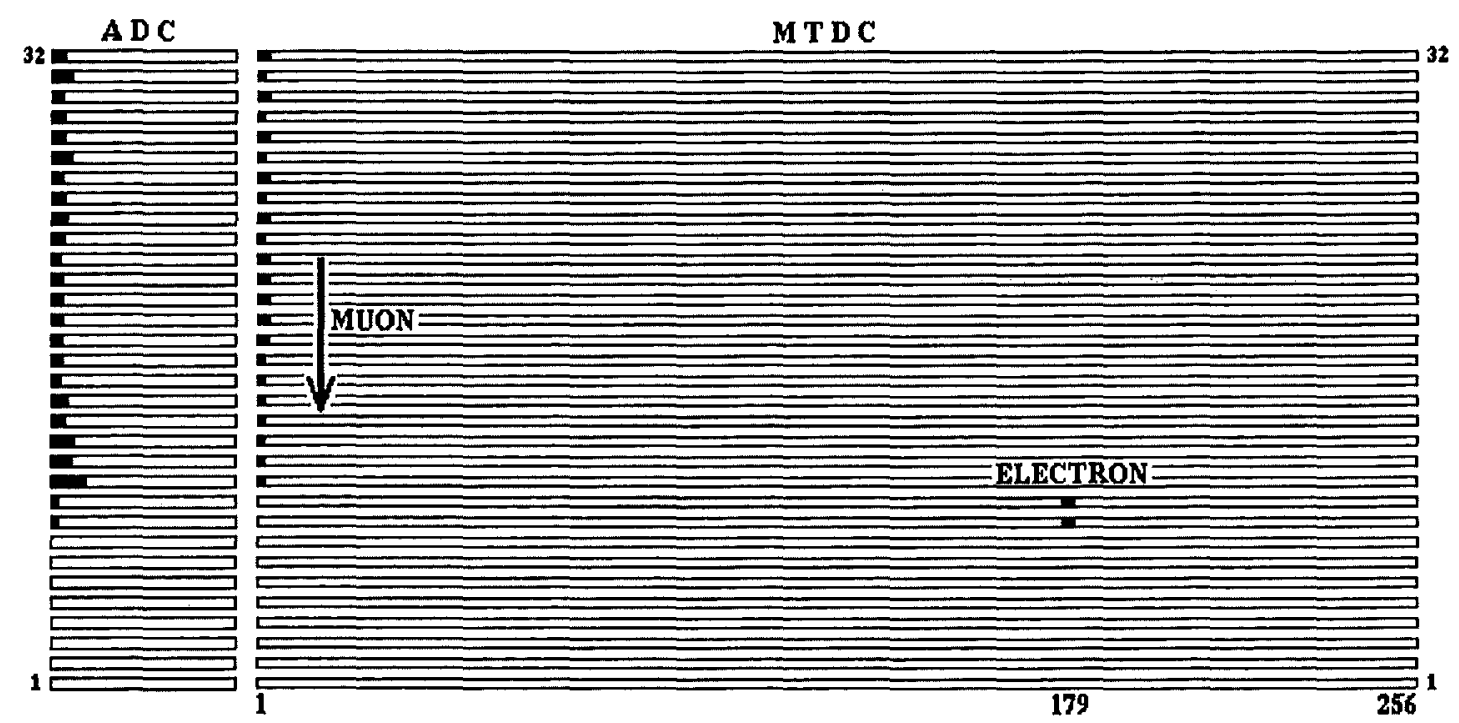

Figure 5: Event display: ADC and MTDC image of a muon decay event. The ADC scale corresponds to the $0-4095$ channel range and the MTDC comprises 256 bins of $20 \mathrm{~ns}$ width. The muon stops after penetrating $4+11$ active layers and the electron is seen in the next layer.

A linear time scale is supposed, assuming all bins have equal $20 \mathrm{~ns}$ widths. Nevertheless, a Fourier analysis of this time spectrum revealed some asynchronous behavior between the clock and the address counter (some output bits switched slightly delayed). This is reflected in periodical discontinuities of the time spectrum at channel numbers of powers of 2. Therefore, before any detailed investigation, the 256 channels were grouped every two bins and the result with $40 \mathrm{~ns}$ per bin was used further for the data analysis.

\subsection{Detector efficiency calculations}

Simulations show that the decay electrons deposit mainly low energy in the scintillator (see Fig. 8a), thus a threshold around few $\mathrm{MeV}$ will significantly affect their detection efficiency. This would subsequently influence the calculation of the four detector constants and finally lead to a wrong value of the charge ratio. Therefore, in order to perform realistic MC simulations, the shape of the energy thresholds must be precisely calibrated. 


\subsubsection{Energy calibration of the active layers}

For the calibration procedure cosmic ray muons are used that traverse all the 20 modules of the calorimeter (the first and the last layer PM were used as trigger). The data are based upon 600000 events and are obtained in an acquisition time of 21 hours. Fig. 6a,b show the two PM anode spectra for one of the scintillator plates. In order to compensate the different amplifications along the electronic chain, all anode spectra are normalized. Due to the absorption of the light in the relatively large scintillator, the signals on both anodes of one plate become position sensitive. This is reflected in the ratio of the anode signal amplitudes for one scintillator which shows a spectrum of values as in Fig. 6c.
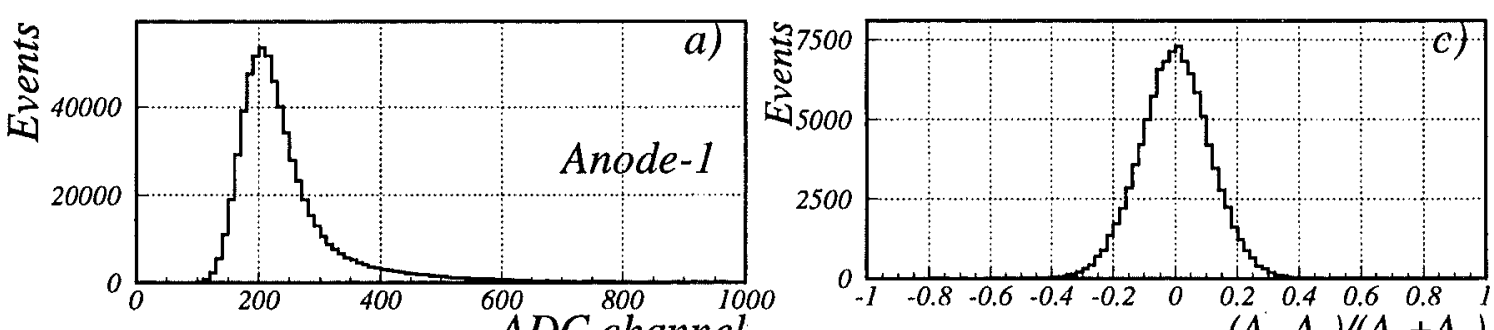

ADC channel
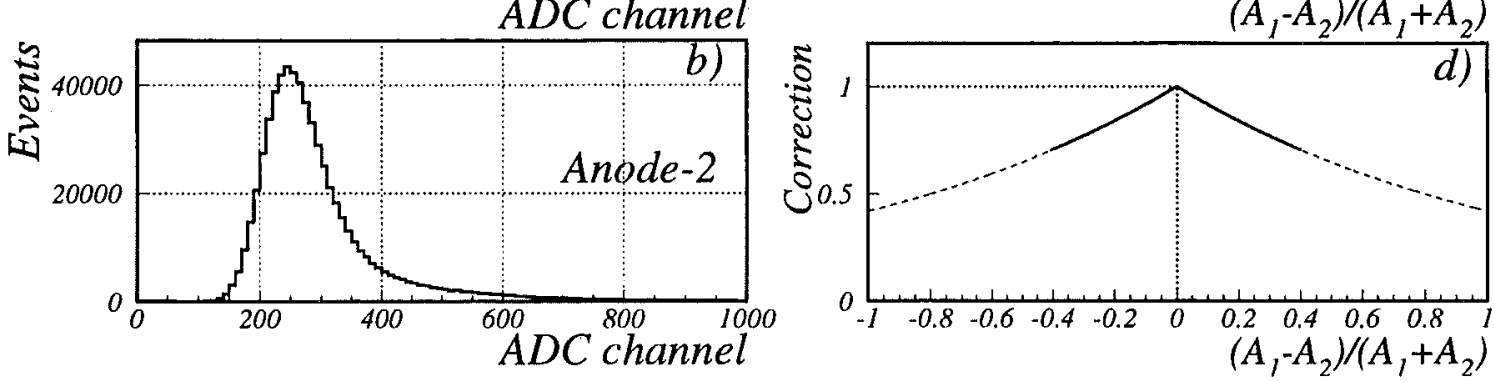

Figure 6: Single anode spectra from calibration measurements for one scintillator plate $(a, b)$; the ratio between the difference and the sum of the two normalized anode signals of one scintillator plate (c); correction factor used in eq. 6 (d).

Simulations have been done with muons with energies higher than $800 \mathrm{MeV}$ impinging on top of the instrument, using the energy spectrum data from [20] and taking into account a distribution of the zenith angle $I(\Theta)=I\left(0^{\circ}\right) \cos ^{2} \Theta$ and uniform in the azimuth angle $\Phi$. At these energies the muons can be considered minimum ionizing particles. The most probable energy loss of a muon crossing vertically the $3 \mathrm{~cm}$ of scintillator is $6 \mathrm{MeV}$. The maximum in the angular distribution of the accepted muon flux is around $20^{\circ}$ zenith angle, thus the effective scintillator thickness crossed by the particle leads to $6.4 \mathrm{MeV}$ as most probable energy deposit. A maximum zenith angle for accepted events of $60^{\circ}$ (with a low probability) corresponds to $12 \mathrm{MeV}$.

By comparing the data with the MC simulations, the energy calibration for the anode signals can be investigated. The energy deposit per event can be assumed as the sum of the normalized anode signals, $\mathrm{A}_{1}$ and $\mathrm{A}_{2}$, multiplied by an exponential correction for the dependence on the impact point 


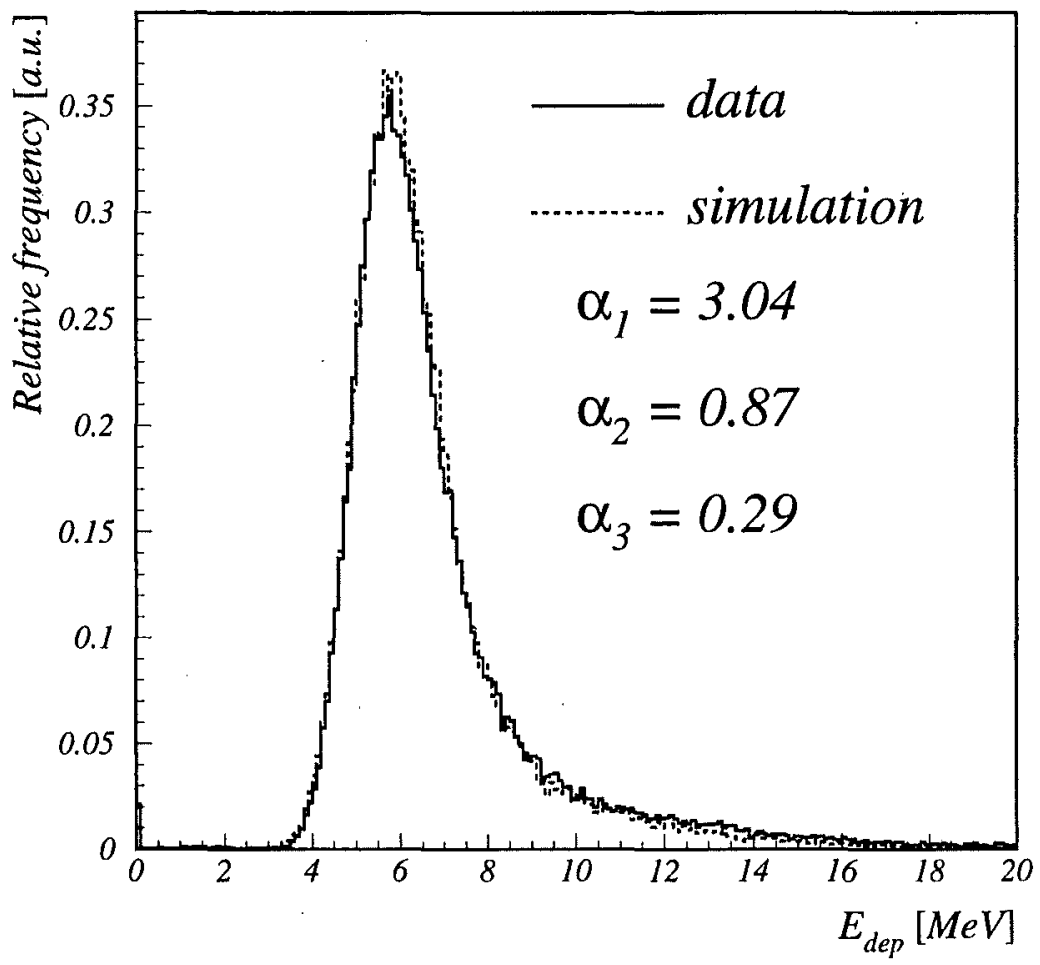

Figure 7: Calibration results for a single plate: energy deposit spectra and parameters in eq. 6 and 7 .

$$
E[M e V]=\alpha_{1}\left(A_{1}+A_{2}\right) \exp \left(-\alpha_{2}\left|\frac{A_{1}-A_{2}}{A_{1}+A_{2}}\right|\right)
$$

where the experimental resolution is taken into account by

$$
\sigma_{E}=\alpha_{3} \sqrt{E[M e V]}
$$

The three parameters, $\alpha_{1}, \alpha_{2}$ and $\alpha_{3}$, have been determined for each scintillator plate by minimizing the difference between the data and the simulation spectra. The good agreement (Fig. 7) justifies the assumption made for the calibration.

\subsubsection{Energy deposit thresholds for the MTDC events}

The thresholds in the energy deposit for the measurements with the MTDC system are not very sharp. They can be determined by comparing data with simulation at low energy deposit. This occurs when only the decay electron is detected in the scintillator (see Fig. 5). Spectra of the energy deposit up to $10 \mathrm{MeV}$ are built with such data (Fig. 8a), where the effect of a threshold can be noticed when comparing with the simulations (Fig. 8b), where no thresholds are considered. The observed experimental excess of events at very low energy has to be accounted as uncorrelated ADC and MTDC information. 

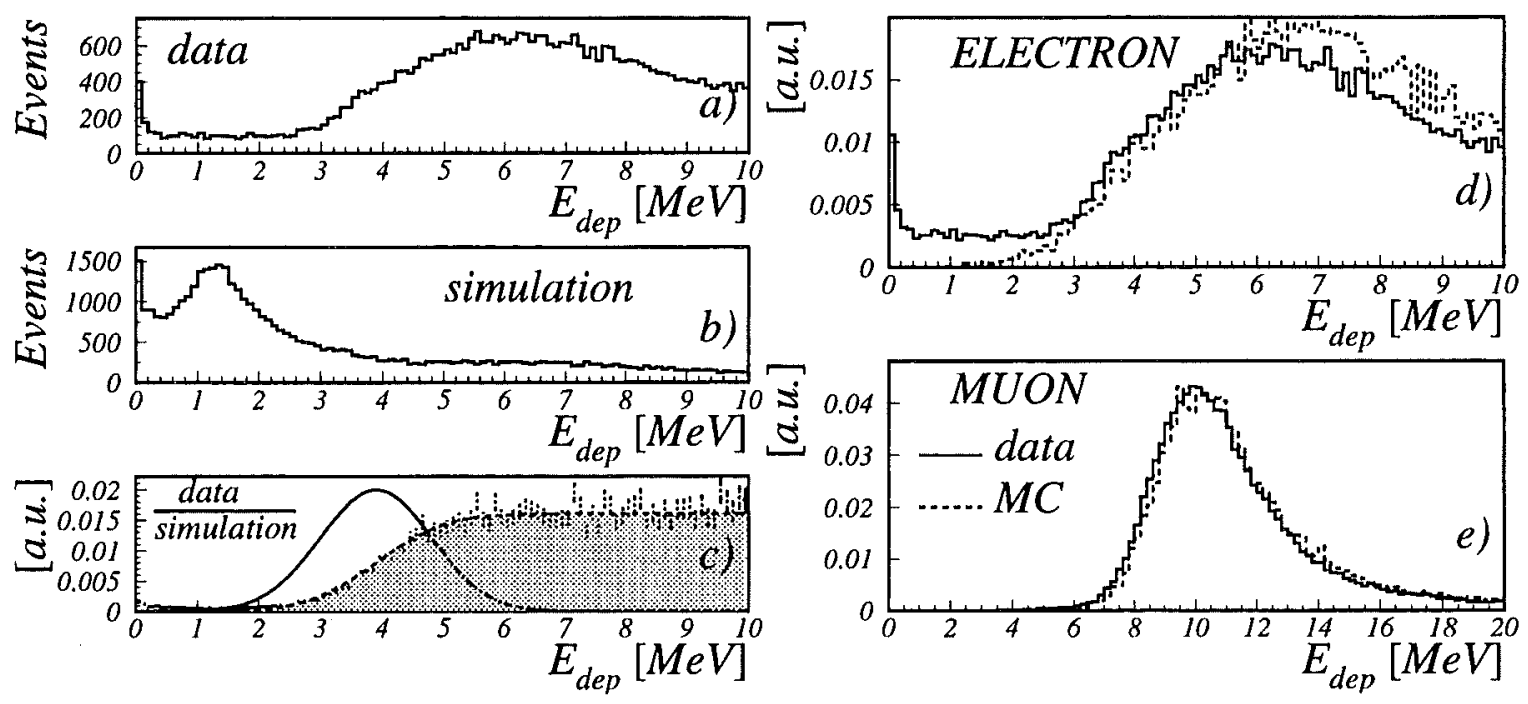

Figure 8: Shape determination of the threshold of a single active layer: spectra of the energy deposit in the scintillator from data (a), simulation (b) and their ratio (scattered points in (c)); a Gauss-form (continuous line) gives the normal frequency function (grey area) to approximate the efficiency function in the scintillator; the parameters are $\bar{E}_{t h r}=$ $3.9 \mathrm{MeV}$ and $\sigma_{t h r}=0.9 \mathrm{MeV}$.

By dividing the two spectra, the shape of the diffuse threshold is estimated (Fig. 8c). It can be seen that the efficiency function is well approximated by a normal frequency function. The deduced threshold shapes are introduced in the simulations and the spectrum of the energy deposited by the decay electrons is compared with the data (Fig. 8d). In Fig. 8e the spectra of the energy deposited by the muons at the end of their track ("stopping layer") are compared too.

\section{Discussion and results}

In Fig. 9a the detector efficiency constants $c_{0}$ and $c_{i}^{*}, i=1,2,3$ from eq. 4, determined by the MC simulations, are used to illustrate the four exponentials which have to be deconvoluted from the total decay curve. Instead of the two muon numbers the total muon number

$$
N_{0}=N_{+}+N_{-}
$$

and the charge ratio

$$
R\left(\mu^{+} / \mu^{-}\right)=\frac{N^{+}}{N^{-}}
$$

are used, thus eq. 4 is written 

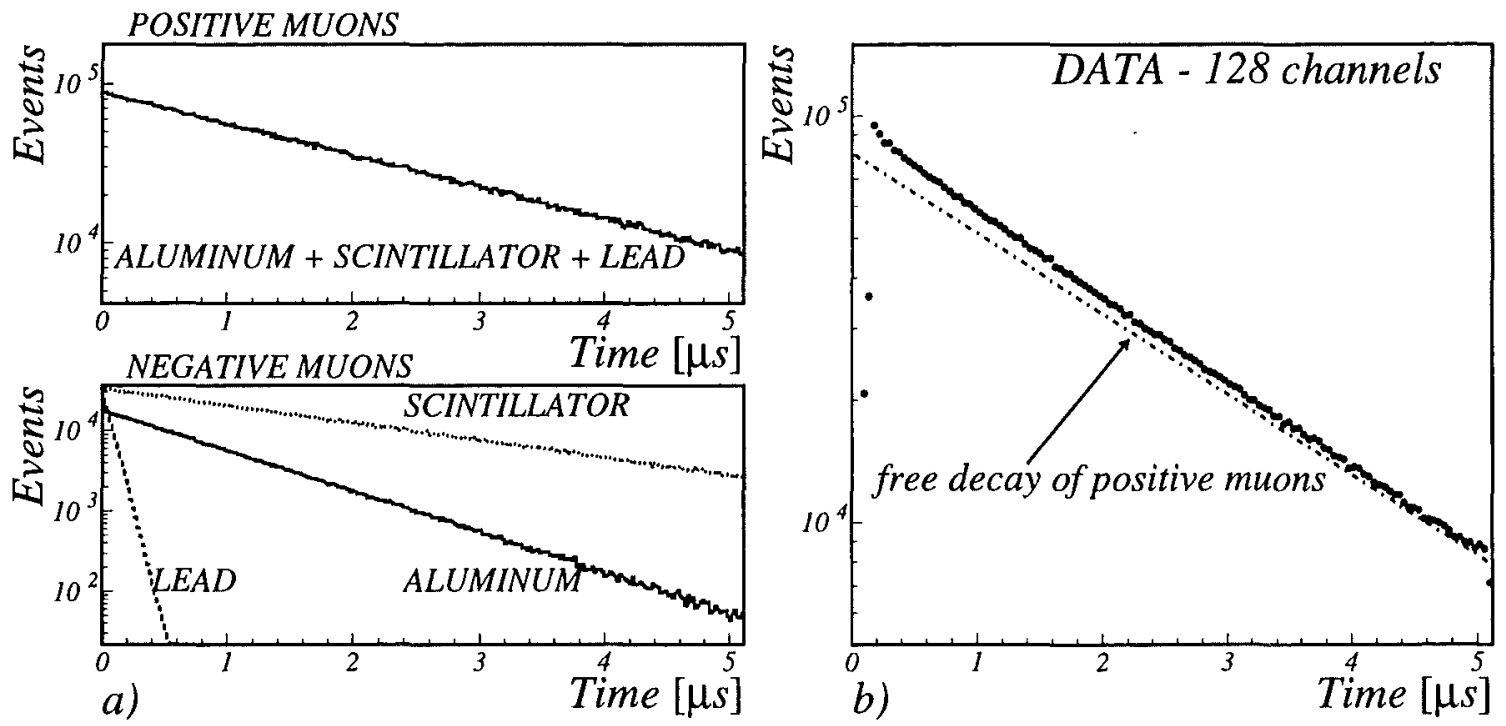

Figure 9: MC simulation results on different materials contribution to the total decay curve (a); Experimental data decay curve (b).

$$
\Delta N\left(t_{i}, t_{i}+\Delta t\right)=\frac{N_{0}}{R+1}\left\{R c_{0} \delta_{0} \exp \left(-\frac{t_{i}}{\tau_{0}}\right)+\left[\sum_{j=1}^{3} c_{j}^{*} \delta_{j} \exp \left(-\frac{t_{i}}{\tau_{j}}\right)\right]\right\} .
$$

Fig. 9b displays experimental data for about 5.3 million events. The first 10 channels could not be evaluated mainly because of the $80 \mathrm{~ns}$ second trigger condition. The free decay curve ( $\tau=2.19703 \mu \mathrm{s}$, adequately normalized) is plotted for comparison.

The background from random coincidences in the cosmic ray flux, described in Sec. 3.1, follows an exponential which is superimposed to the decay spectrum. Nevertheless, due to the low rate, this background is practically uniformly distributed in the time window of $5.12 \mu \mathrm{s}$.

In particular, the background has been considered in the data analysis as follows. Although the life time of the muons is recorded from $\approx 0$. to $5.12 \mu$ s there should be no systematic difference when fitting the decay curve only in parts of the time range (see also [16]). For instance, one could fix the end point of the fit at the end of the 128 channels in the time spectrum and shift the first point of the fit in the positive direction of the time axis, starting from channel 10 (400 ns). Without background subtraction, a systematic increase in the charge ratio $R\left(\mu^{+} / \mu^{-}\right)$is noticed. This can be related to the decreasing "signal/noise" ratio. A constant background (slope zero) in all the time range will force the fit to "look for" those components which participate in the total spectrum with smaller slopes, therefore the number of positive muons (largest mean life time, i.e. smallest slope) will be overestimated compared to that of negative muons. The "stability" over different fit intervals has been the criterion for choosing the value of the constant background. This was found to be 110 events per time bin, in good agreement with a 

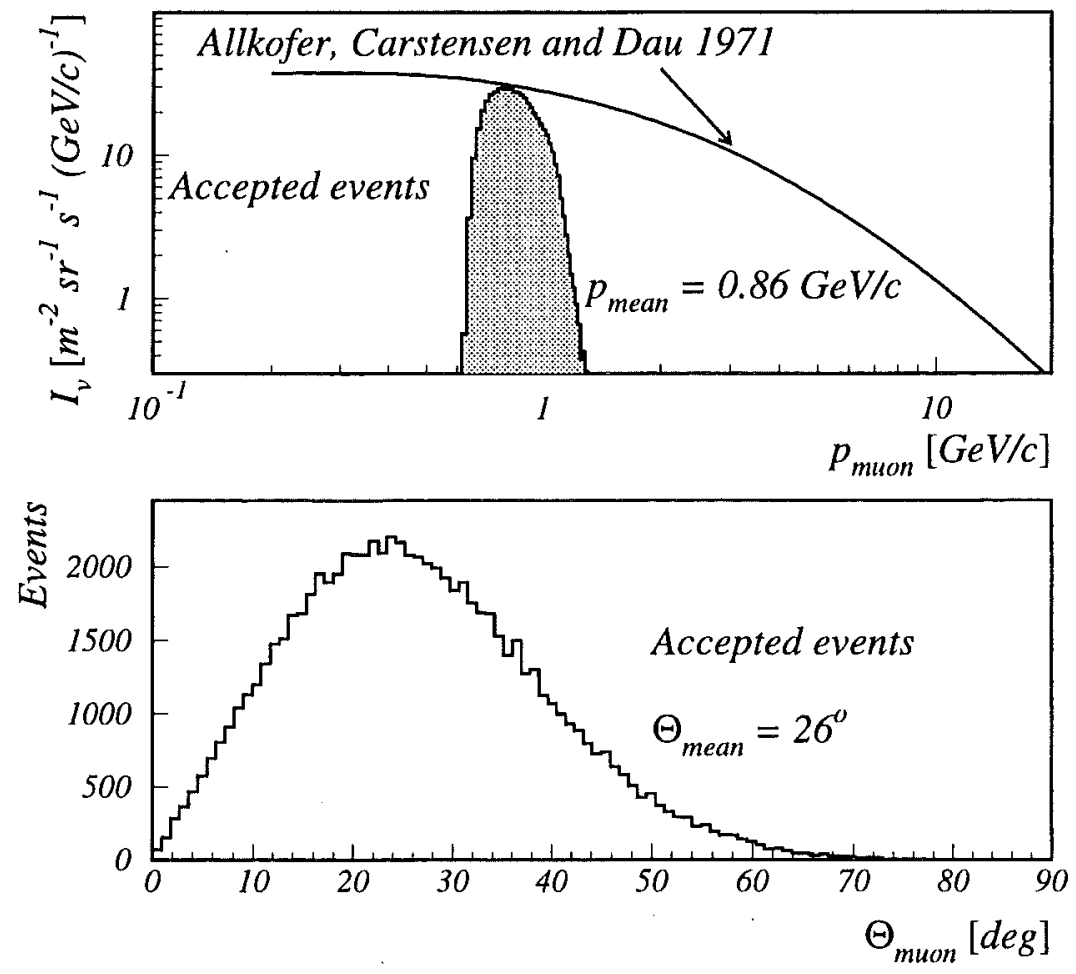

Figure 10: Momentum and zenith angle distributions of accepted events. The measured intensity of the vertical muon flux is shown for comparison (Allkofer, Carstensen and Dau $[20])$.

measurement done up to $22 \mu$ s in which the background could be seen in the last bins separated from the decay exponentials. The background was afterwards subtracted from the data before fitting the two numbers, $N_{0}$ and $R\left(\mu^{+} / \mu^{-}\right)$.

The present measurement results in $R\left(\mu^{+} / \mu^{-}\right)=1.30 \pm 0.05$ (Fig. 11), a value slightly different from the preliminary measurement with only 10 detector modules [21]. From the $\mathrm{MC}$ simulations it is estimated that the muon momentum is between 0.6 and $1.2 \mathrm{GeV} / \mathrm{c}$ with a mean value of $0.86 \mathrm{GeV} / \mathrm{c}$ and the mean zenith angle is $26^{\circ}$. The error in $\mathrm{R}$ is that of the corresponding parameter in the fit procedure. Fig. 9b illustrates the quality of the data. With sufficient statistics the difference between the data and the positive muon decay curve is clearly visible. A decrease of this quality (by lowering the statistics) makes the result of the fit more dependent on the fit conditions, i.e. the first affected is the "stability" discussed above. 


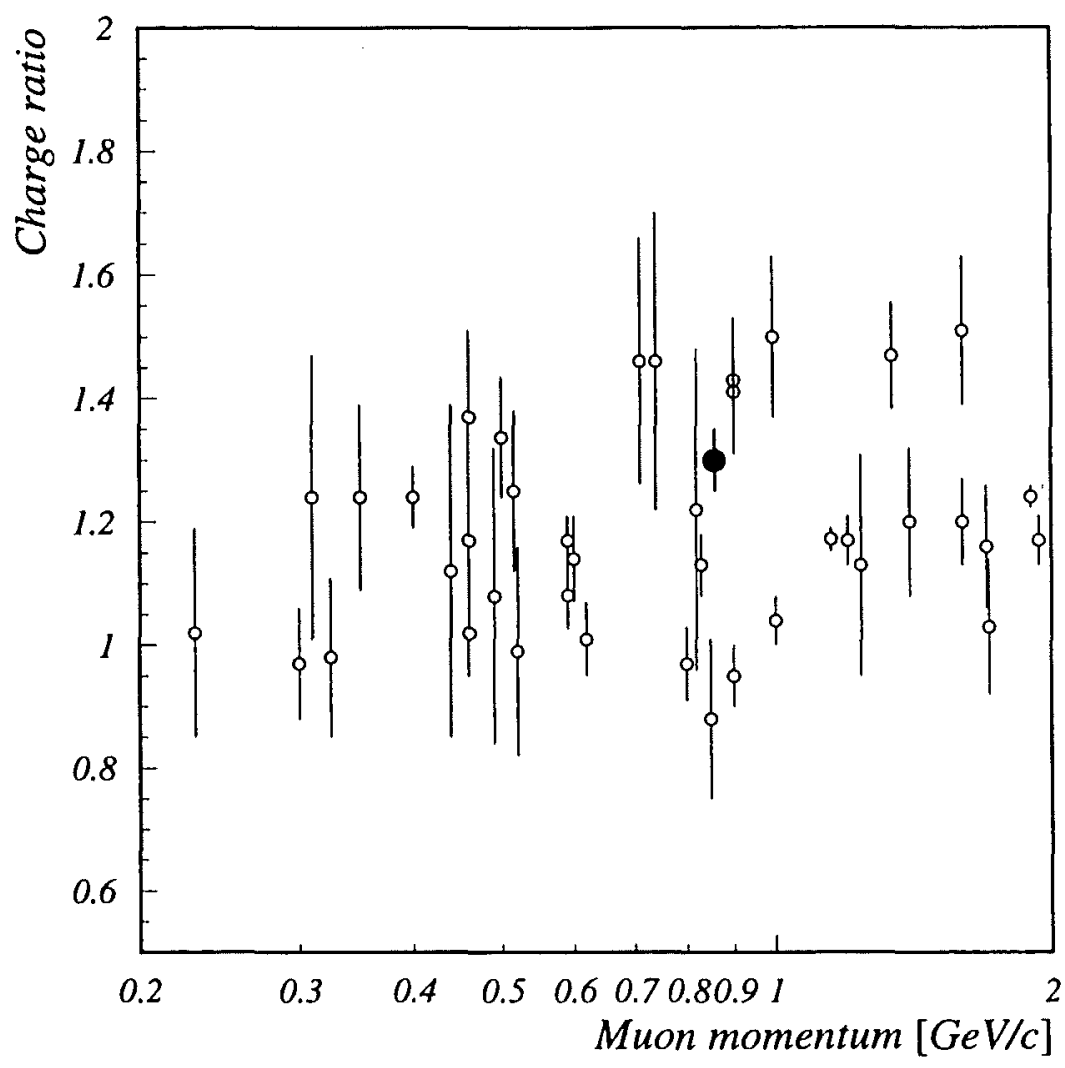

Figure 11: Selected results from Fig. 1 for muon momentum $<2 \mathrm{GeV} / \mathrm{c}$. $\bullet-$ present result at mean momentum $0.86 \mathrm{GeV} / \mathrm{c}$.

\section{Conclusions}

A compact detector has been set up by applying a modified method of delayed coincidences to determine the charge ratio of cosmic ray muons below $1 \mathrm{GeV} / \mathrm{c}$, using a configuration initially designed for prototype studies of high energy muon spectrometry [22]. The aluminum as absorber material leads for negative muons to a significant difference in the mean life time compared to that of the free decay of positive muons, detectable with simple time electronics and sufficiently high rates. A first measurement of the charge ratio of positive to negative muons with a mean momentum of $0.86 \mathrm{GeV} / \mathrm{c}$ and a mean zenith angle of $26^{\circ}$ gives $1.30 \pm 0.05$ and demonstrates the capability of the detector.

The method overcomes the difficulties of magnetic spectrometer experiments with a better discrimination of muons by the detection of their decay electrons. The background can be further reduced when surrounding the detector modules with anti-counters. This is important for telescope measurements with inclinations, where the muon flux will drop while the background increases. Due to the original experimental purpose the WILLI setup contains lead absorbers. The lead, however, plays no significant role for the present 
studies, but it reduces the contribution of aluminum by absorbing low energy decay electrons. It is also possible to extend the measurement of the decay exponentials into a region were the separation could be better, as long as the required statistics remains reasonable. Such a modified version is now under construction for systematic measurements of the charge ratio of the inclusive muon flux with different conditions.

\section{Acknowledgements}

The authors affiliated to the National Institute of Physics and Nuclear Engineering, Bucharest, are grateful for the considerable support received from the International Bureau Bonn (WTZ - Project) and Institut für Kernphysik, Forschungszentrum Karlsruhe (FZK) for setting up the electromagnetic calorimeter. One of us (B. V.) would like to thank for the hospitality during his research visit in FZK, when a large part of the analysis and the $\mathrm{MC}$ simulations have been done in the frame of a dedicated project of the Volkswagen Foundation. We thank Prof. D. Müller from the University of Chicago for useful discussions about the manuscript. Finally we thank Mrs. Laura Vulpescu for her help in preparing the figures.

\section{References}

[1] J. L. Morrison and J. W. Elbert, Proc. of the $13^{\text {th }}$ ICRC 3 (1973) 1833.

[2] A. Goned et al., Proc. of the $19^{\text {th }}$ ICRC 8 (1985) 192.

[3] H. M. Portella et al., Proc. of the $23^{\text {rd }}$ ICRC 4 (1993) 464.

[4] T. K. Gaisser, T. Stanev and G. Barr, Proc. of the $20^{\text {th }}$ ICRC 6 (1987) 169.

[5] P. Lipari, Astropart. Phys. 1 (1995) 195.

[6] M. Honda et al., Phys. Rev. D 52 (1995) 4985.

[7] H. Jones, Rev. Mod. Phys. 11 (1939) 235.

[8] G. Basini et al., Proc. of the $24^{\text {th }}$ ICRC 1 (1995) 585.

[9] J. F. Krizmanic et al., Proc. of the $24^{\text {th }}$ ICRC 1 (1995) 593.

[10] G. Tarle et al., Proc. of the $25^{\text {th }}$ ICRC 6 (1997) 321.

[11] M. Conversi, Phys. Rev. 79 (1950) 749.

[12] K. P. Singhal, Proc. of the $18^{\text {th }}$ ICRC 7 (1983) 27.

[13] N. C. Mukhopadhyay, Phys. Lett. C 30 (1977) 1. 
[14] T. Suzuki, D.F Measday and J.P. Roalsvig, Phys. Rev. C 35 (1987) 2212.

[15] M. Yamada et al., Phys. Rev. D 44 (1991) 617.

[16] W. Grandegger, KfK Report 5122, Kernforschungszentrum Karlsruhe (1993)

[17] T. E. Jannakos, FZK Report 5520, Forschungszentrum Karlsruhe (1995).

[18] J. A. Wheeler, Mod. Phys. 21 (1949) 133.

[19] CERN Program Library Long Writeup W5013, (1994).

[20] O. C. Allkofer, K. Carstensen and W. D. Dau, Phys. Lett. B 36 (1971) 425.

[21] B. Vulpescu, J. Wentz, I. M. Brâncus, H. Rebel, A. F. Badea, H. Bozdog, M. Duma, A. Haungs, H.-J. Mathes and M. Petcu, Nucl. Phys. B (Proc. Suppl.) 52 (1997) 195.

[22] I. M. Brâncuş et al., Balk. Phys. Lett. 4 (1996) 54.

[23] D. F. Smart and M. A. Shea, Proc. of the $25^{\text {th }}$ ICRC 2 (1997) 401.

[24] B. G. Owen and J. G. Wilson, Proc. Phys. Soc. (London) A 64 (1951) 417.

[25] J. R. Moroney and J. K. Parry, Austral. Journ. Phys. 7 (1954) 424.

[26] S. Fukui, Journ. Phys. Soc. Jap. 10 (1955) 735.

[27] J. E. R. Holmes, B. G. Owen and A. L. Rodgers, Proc. Phys. Soc. (London) 78 (1961) 505.

[28] P. J. Hayman and A. W. Wolfendale, Nature 195 (1962) 166.

[29] D. W. Coates and W. F. Nash, Austral. Journ. Phys. 15 (1962) 420.

[30] R. J. R. Judge and W. F. Nash, Nuovo Cimento 35 (1965) 999.

[31] B. C. Rastin, S. R. Baber, R. M. Bull and W. F. Nash, Proc. of the $9^{\text {th }}$ ICRC 2 (1965) 981.

[32] S. Kawaguchi, T. Sakai, H. Oda, H. Ueno and Y. Kamiya, Proc. of the $9^{\text {th }}$ ICRC 2 (1965) 941.

[33] O. C. Allkofer and R. D. Andresen, Nuovo Cimento 51 (1967) 329.

[34] O. C. Allkofer, R. D. Andresen and W. D. Dau, Can. Journ. Phys. 46 (1968) S301.

[35] S. R. Baber, W. F. Nash and B. C. Rastin, Nucl. Phys. B 4 (1968) 549.

[36] O. C. Allkofer and K. Klausen, Acta. Phys. Acad. Scien. Hun. 29 Suppl. 2 (1969) 698. 
[37] I. C. Appleton, M. T. Hogue and B. C. Rastin, Nucl. Phys. B 26 (1971) 365.

[38] O. C. Allkofer and W. D. Dau, Phys. Lett B 38 (1972) 439.

[39] T. H. Burnett, L. J. LaMay, G. E. Masek and T. Maung, Phys. Rev. Lett. 30 (1973) 937.

[40] T. H. Burnett, G. E. Masek, T. Maung, E. S. Miller, H. Ruderman and W. Vernon, Proc. of the $13^{\text {th }}$ ICRC 3 (1973) 1764.

[41] M. S. Abdel-Monem, J. R. Benbrook, A. R. Osborne and W. R. Sheldon, Proc. of the $13^{\text {th }}$ ICRC 3 (1973) 1811.

[42] B. J. Bateman, N. M. Duller, P. J. Green and A. V. Jelinek, Proc. of the $13^{\text {th }}$ ICRC 3 (1973) 1816.

[43] M. G. Thompson et al., Proc. of the $13^{\text {th }}$ ICRC 3 (1973) 1822.

[44] B. C. Rastin, Journ. Phys. G 10 (1984) 1629.

[45] S. A. Stephens R. L. and Golden, Proc. of the $20^{\text {th }}$ ICRC 6 (1987) 173.

[46] G. Basini et al., Proc. of the $22^{\text {nd }}$ ICRC 4 (1991) 544.

[47] E. Schneider et al., Proc. of the $24^{\text {th }}$ ICRC 1 (1995) 690. 


\section{Appendix}

Table 2 compiles experimental results, shown in Fig. 1 and 11, indicating the experimental method used and the reference. The geomagnetic cut-off $\left(R_{c}\right)$ is calculated by interpolating a two dimensional grid (latitude and longitude) for the geographical location of the particular experiment [23].

Table 2: Method: A-delayed coincidences, B - magnetic spectrometer.

\begin{tabular}{|c|c|c|c|c|}
\hline Author/Ref. & $\mathrm{p}_{\mu}^{\text {mean }}[\mathrm{GeV} / \mathrm{c}]$ & $\mathrm{R}\left(\mu^{+} / \mu^{-}\right)$ & Method & $\mathrm{R}_{c}[\mathrm{GV}]$ \\
\hline Conversi $[11]$ & $\overline{0.4}$ & $1.24 \pm 0.05$ & A & 6.4 \\
\hline Conversi [11] & 0.35 & $1.24 \pm 0.15$ & A & 1.9 \\
\hline \multirow[t]{6}{*}{ Owen [24] } & 1.15 & $1.172 \pm 0.019$ & B & 2.9 \\
\hline & 1.90 & $1.241 \pm 0.018$ & & \\
\hline & 2.75 & $1.251 \pm 0.018$ & & \\
\hline & 4.90 & $1.263 \pm 0.019$ & & \\
\hline & 6.76 & $1.240 \pm 0.023$ & & \\
\hline & 11.48 & $1.229 \pm 0.036$ & & \\
\hline \multirow[t]{7}{*}{ Moroney [25] } & 0.6 & $1.14 \pm 0.07$ & B & 3.0 \\
\hline & 1.6 & $1.20 \pm 0.07$ & & \\
\hline & 1.7 & $1.16 \pm 0.10$ & & \\
\hline & 2.6 & $1.39 \pm 0.12$ & & \\
\hline & 3.4 & $1.28 \pm 0.08$ & & \\
\hline & 4.3 & $1.36 \pm 0.12$ & & \\
\hline & 9.0 & $1.38 \pm 0.12$ & & \\
\hline \multirow[t]{4}{*}{ Fukui [26] } & 0.85 & $0.88 \pm 0.13$ & B & 12.2 \\
\hline & 1.71 & $1.03 \pm 0.11$ & & \\
\hline & 3.72 & $1.27 \pm 0.14$ & & \\
\hline & 10. & $1.27 \pm 0.15$ & & \\
\hline \multirow[t]{3}{*}{ Holmes [27] } & 6.7 & $1.39 \pm 0.08$ & B & 2.9 \\
\hline & 11. & $1.35 \pm 0.08$ & & \\
\hline & 18. & $1.29 \pm 0.08$ & & \\
\hline \multirow[t]{3}{*}{ Hayman [28] } & 6.7 & $1.229 \pm 0.049$ & B & 2.1 \\
\hline & 10.4 & $1.223 \pm 0.038$ & & \\
\hline & 17.5 & $1.233 \pm 0.037$ & & \\
\hline
\end{tabular}


(continuation)

\begin{tabular}{|c|c|c|c|c|}
\hline Author/Ref. & $\mathrm{p}_{\mu}^{\text {mean }}[\mathrm{GeV} / \mathrm{c}]$ & $\mathrm{R}\left(\mu_{.}^{+} / \mu^{-}\right)$ & Method & $\mathrm{R}_{c}[\mathrm{GV}]$ \\
\hline Coates [29] & 4.32 & $1.33 \pm 0.10$ & $\mathrm{~B}$ & 2.6 \\
\hline Rastin [31] & 4.3 & $1.217 \pm 0.033$ & B & 2.6 \\
\hline \multirow[t]{3}{*}{ Kawaguchi [32] } & 7.4 & $1.284 \pm 0.094$ & B & 12.0 \\
\hline & 10.6 & $1.249 \pm 0.076$ & & \\
\hline & 15.3 & $1.312 \pm 0.068$ & & \\
\hline \multirow[t]{9}{*}{ Allkofer [34] } & 0.325 & $0.98 \pm 0.13$ & B & 14.1 \\
\hline & 0.515 & $1.25 \pm 0.13$ & & \\
\hline & 0.71 & $1.46 \pm 0.20$ & & \\
\hline & 0.995 & $1.50 \pm 0.13$ & & \\
\hline & 1.4 & $1.20 \pm 0.12$ & & \\
\hline & 2.42 & $1.64 \pm 0.12$ & & \\
\hline & 5.4 & $1.32 \pm 0.12$ & & \\
\hline & 13. & $0.95 \pm 0.14$ & & \\
\hline & 19. & $1.07 \pm 0.18$ & & \\
\hline \multirow[t]{5}{*}{ Baber [35] } & 4.0 & $1.22 \pm 0.04$ & B & 2.6 \\
\hline & 6.1 & $1.27 \pm 0.04$ & & \\
\hline & 9.2 & $1.26 \pm 0.06$ & & \\
\hline & 12.4 & $1.25 \pm 0.08$ & & \\
\hline & 17.1 & $1.27 \pm 0.09$ & & \\
\hline \multirow[t]{5}{*}{ Allkofer $[36]$} & 0.5 & $1.337 \pm 0.098$ & $\mathrm{~B}$ & 2.3 \\
\hline & 1.34 & $1.469 \pm 0.087$ & & \\
\hline & 2.67 & $1.606 \pm 0.130$ & & \\
\hline & 4.57 & $1.364 \pm 0.131$ & & \\
\hline & 8.31 & $1.275 \pm 0.140$ & & \\
\hline \multirow[t]{5}{*}{ Appleton [37] } & 3.8 & $1.249 \pm 0.024$ & $\mathrm{~B}$ & 2.6 \\
\hline & 6. & $1.229 \pm 0.020$ & & \\
\hline & 9.2 & $1.256 \pm 0.027$ & & \\
\hline & 12.7 & $1.312 \pm 0.039$ & & \\
\hline & 17.2 & $1.263 \pm 0.038$ & & \\
\hline \multirow[t]{2}{*}{ Allkofer [38] } & 0.46 & $1.17 \pm 0.11$ & $\mathrm{~B}$ & 14.1 \\
\hline & 0.90 & $1.43 \pm 0.10$ & & \\
\hline
\end{tabular}


(continuation)

\begin{tabular}{|c|c|c|c|c|}
\hline Author/Ref. & $\mathrm{p}_{\mu}^{\text {mean }}[\mathrm{GeV} / \mathrm{c}]$ & $\mathrm{R}\left(\mu^{+} / \mu^{-}\right)$ & Method & $\mathrm{R}_{c}[\mathrm{GV}]$ \\
\hline & 1.60 & $1.51 \pm 0.10$ & & \\
\hline & 2.40 & $1.71 \pm 0.14$ & & \\
\hline & 3.80 & $1.36 \pm 0.10$ & & \\
\hline & 11.40 & $1.06 \pm 0.07$ & & \\
\hline & 0.46 & $1.37 \pm 0.14$ & & 2.3 \\
\hline & 0.90 & $1.41 \pm 0.10$ & & \\
\hline & 1.60 & $1.51 \pm 0.12$ & & \\
\hline & 2.40 & $1.66 \pm 0.16$ & & \\
\hline & 3.80 & $1.53 \pm 0.14$ & & \\
\hline & 11.40 & $1.22 \pm 0.10$ & & \\
\hline \multirow[t]{5}{*}{ Abdel-Monem [41] } & 3. & $1.20 \pm 0.06$ & B & 4.8 \\
\hline & 5.3 & $1.24 \pm 0.07$ & & \\
\hline & 8.4 & $1.26 \pm 0.11$ & & \\
\hline & 12.1 & $1.24 \pm 0.12$ & & \\
\hline & 18.8 & $1.24 \pm 0.15$ & & \\
\hline \multirow[t]{7}{*}{ Thompson [43] } & 9.7 & $1.277 \pm 0.008$ & B & 2.1 \\
\hline & 11.9 & $1.252 \pm 0.017$ & & \\
\hline & 12.4 & $1.295 \pm 0.007$ & & \\
\hline & 13.6 & $1.284 \pm 0.014$ & & \\
\hline & 13.9 & $1.286 \pm 0.003$ & & \\
\hline & 16.1 & $1.281 \pm 0.016$ & & \\
\hline & 19.6 & $1.288 \pm 0.015$ & & \\
\hline \multirow[t]{2}{*}{ Burnett [39] } & 14.4 & $1.32 \pm 0.05$ & B & 5.8 \\
\hline & 17.4 & $1.25 \pm 0.05$ & & \\
\hline \multirow[t]{4}{*}{ Singhal [12] } & 0.23 & $1.02 \pm 0.17$ & A & 15.6 \\
\hline & 0.31 & $1.24 \pm 0.23$ & & \\
\hline & 0.52 & $0.99 \pm 0.17$ & & \\
\hline & 0.74 & $1.46 \pm 0.24$ & & \\
\hline \multirow[t]{3}{*}{ Rastin [44] } & 4.48 & $1.361 \pm 0.034$ & $B$ & 2.6 \\
\hline & 4.96 & $1.336 \pm 0.026$ & & \\
\hline & 5.37 & $1.322 \pm 0.025$ & & \\
\hline
\end{tabular}


(continuation)

\begin{tabular}{|c|c|c|c|c|}
\hline Author/Ref. & $\mathrm{p}_{\mu}^{\text {mean }}[\mathrm{GeV} / \mathrm{c}]$ & $\mathrm{R}\left(\mu^{+} / \mu^{-}\right)$ & Method & $\overline{\mathrm{R}_{c}[\mathrm{GV}]}$ \\
\hline & 5.67 & $1.336 \pm 0.031$ & & \\
\hline & 5.91 & $1.320 \pm 0.028$ & & \\
\hline & 6.18 & $1.308 \pm 0.026$ & & \\
\hline & 6.49 & $1.302 \pm 0.024$ & & \\
\hline & 6.84 & $1.310 \pm 0.023$ & & \\
\hline & 7.24 & $1.231 \pm 0.020$ & & \\
\hline & 7.70 & $1.271 \pm 0.020$ & & \\
\hline & 8.25 & $1.257 \pm 0.019$ & & \\
\hline & 8.91 & $1.257 \pm 0.018$ & & \\
\hline & 9.70 & $1.249 \pm 0.017$ & & \\
\hline & 10.69 & $1.239 \pm 0.016$ & & \\
\hline & 11.94 & $1.247 \pm 0.016$ & & \\
\hline & 13.58 & $1.251 \pm 0.016$ & & \\
\hline & 15.81 & $1.285 \pm 0.016$ & & \\
\hline & 19.05 & $1.263 \pm 0.016$ & & \\
\hline \multirow[t]{11}{*}{ Stephens [45] } & 0.8 & $0.97 \pm 0.06$ & B & 4.9 \\
\hline & 0.9 & $0.95 \pm 0.05$ & & \\
\hline & 1.0 & $1.04 \pm 0.04$ & & \\
\hline & 2.0 & $1.12 \pm 0.03$ & & \\
\hline & 3.0 & $1.18 \pm 0.03$ & & \\
\hline & 8.0 & $1.27 \pm 0.03$ & & \\
\hline & 10. & $1.44 \pm 0.07$ & & \\
\hline & 11.2 & $1.26 \pm 0.07$ & & \\
\hline & 13.0 & $1.17 \pm 0.06$ & & \\
\hline & 15.3 & $1.23 \pm 0.05$ & & \\
\hline & 18.1 & $1.14 \pm 0.04$ & & \\
\hline \multirow[t]{5}{*}{ Basini [46] } & 0.3 & $0.97 \pm 0.09$ & B & 0.7 \\
\hline & 0.46 & $1.02 \pm 0.07$ & & \\
\hline & 0.62 & $1.01 \pm 0.06$ & & \\
\hline & 0.83 & $1.13 \pm 0.05$ & & \\
\hline & 1.2 & $1.17 \pm 0.04$ & & \\
\hline
\end{tabular}


(continuation)

\begin{tabular}{lcccc}
\hline Author/Ref. & $\mathrm{p}_{\mu}^{\text {mean }}[\mathrm{GeV} / \mathrm{c}]$ & $\mathrm{R}\left(\mu^{+} / \mu^{-}\right)$ & Method & $\mathrm{R}_{\mathrm{c}}[\mathrm{GV}]$ \\
\hline & 1.94 & $1.17 \pm 0.04$ & & \\
& 3.5 & $1.22 \pm 0.03$ & & \\
& 8.0 & $1.18 \pm 0.03$ & & \\
Grandegger [16] & 5.1 & $1.32 \pm 0.04$ & $\mathrm{~A}$ & 2.9 \\
Jannakos [17] & 5.1 & $1.28 \pm 0.02$ & $\mathrm{~A}$ & 2.9 \\
Basini [8] & 0.49 & $1.08 \pm 0.24$ & $\mathrm{~B}$ & 4.3 \\
& 0.82 & $1.22 \pm 0.26$ & & \\
Krizmanic [9] & 1.24 & $1.13 \pm 0.18$ & & \\
Schneider [47] & 0.44 & $1.12 \pm 0.27$ & $\mathrm{~B}$ & 0.4 \\
Tarle [10] & 0.59 & $1.17 \pm 0.04$ & $\mathrm{~B}$ & 4.3 \\
WILLI, this work & 0.59 & $1.081 \pm 0.054$ & $\mathrm{~B}$ & 0.4 \\
\hline
\end{tabular}

